\title{
Stiffness Transfer Matrix Method (STMM) for Stable Dispersion Curves Solution in Anisotropic Composites
}

\author{
Ayman Kamal, Victor Giurgiutiu \\ kamal@email.sc.edu, victorg@sc.edu \\ Mechanical Engineering Department, University of South Carolina, Columbia SC, 29208
}

\begin{abstract}
This paper discusses combined transfer matrix method (TMM) with stiffness matrix method (SMM) for obtaining a stable solution for dispersion curves of Lamb wave propagation in non-isotropic layers. TMM developed by Thomson and Haskell experiences numerical deficiency at high frequency thickness simulations. SMM was proposed by different researchers to solve the instability issue of TMM. This study shows that stable SMM is good at high frequencies, and TMM needs to be combined with SMM to obtain stable and robust behavior over the frequency range. Numerical simulations of dispersion curves are presented for wave propagation in orthotropic unidirectional fiber composites and cross ply composites. The paper ends with conclusions and future work.

Keywords: Dispersion curves; instability; stiffness matrix method (SMM); transfer matrix method (TMM); ultrasonics; Lamb waves; Christoffel equation; anisotropic composites
\end{abstract}

\section{INTRODUCTION}

The use of composite materials is currently implemented in many structural components, including automotive parts, civil infrastructures, compensatory devices, and aerospace structures. Composite materials combine the properties of two or more constituent materials. For example, carbon-fiber reinforced polymer composites (CFRP) combine the specific stiffness and strength of carbon fibers with the properties of epoxy matrix. Composite materials can be generally manufactured from metallic, polymeric, or ceramic matrix; however, in this paper the focus is on polymer matrix composites for their wide application in the aerospace industry. Many parts of recent air and spacecraft are manufactured from CFRP and glass-fiber reinforced polymers (GFRP) as well. Because of the challenge of constructing high strength structural parts with constrained light weights, polymer composites are more favorable than metallic alloys. Also, polymer composites can be manufactured into complex shaped components and their properties can be tailored by changing the stacking sequence of layup, i.e. layers or individual lamina.

Detection of damages and flaws as well as structural integrity of polymer composites is receiving as much attention as the advantages and applications of these materials. Lamb waves ultrasonics, or guided plate waves, have long been acknowledged for damage detection in composites $1,2,3,4,5$. For any study of guided waves propagation in structures, wave's propagation speeds are essential for further analysis, e.g. impact source localization, reflection, transmission, and energy and power studies. In many cases, robust predictive models of wave's speeds are needed before conducting experimental studies.

Lamb wave theory is well documented in many references ${ }^{1,6,7,8}$. For isotropic materials, the wave equation can be expressed by two potential functions and the pressure and shear wave velocities. The shear horizontal (SH) wave propagation in this case is decoupled from longitudinal (or pressure waves P) and shear vertical (SV) wave propagations. Lamb waves are symmetric and antisymmetric and they are dispersive by nature, i.e. (are having different speeds at different frequencies). The characteristic equation (Rayleigh-Lamb equation ${ }^{9}$ ) is obtained by solving wave equation and applying stress-free boundary conditions at upper and lower surfaces of the plate.

In the case of fiber reinforced polymer (FRP) composites where the material is generally anisotropic, the three types of guided waves ( $\mathrm{P}, \mathrm{SV}$, and $\mathrm{SH})$ are coupled and it is not possible to separate the dispersion curves of the three types of waves. When dealing with multilayered media, and to be able to solve anisotropic layers, (e.g. composites), it is favorable to use the Christoffel equation which is well documented in references ${ }^{1,10}$. Advanced studies include elastic wave propagation in anisotropic curved plates ${ }^{11}$, and modelling wave propagation in sandwich composites ${ }^{12,13,14}$. There are different methods to calculate dispersion curves in multilayered composite materials (a) Transfer Matrix method (TMM); (b) Global Matrix method (GMM); (c) Semi-Analytical Finite Element method (SAFE); (d) local interaction simulation approach (LISA); and (e) Equivalent Matrix method (EMM) applied on cross-ply cases. In this paper we

Health Monitoring of Structural and Biological Systems 2014, edited by Tribikram Kundu, Proc. of SPIE Vol. 9064, 906410 .

(c) 2014 SPIE · CCC code: 0277-786X/14/\$18 · doi: 10.1117/12.2044789 
review briefly algorithms for calculating dispersion curves in composites and focus on TMM, following references ${ }^{15}, 16$. We show the framework addressed by Schmidt ${ }^{17}$ and Rokhlin $^{18}$ for eliminating the TMM instability by introducing stiffness matrix method (SMM). Eventually, it is shown that TMM and SMM need to be combined together to get the desired stable and accurate results at both low/high frequency-thickness values. Numerical simulations of dispersion curves are presented for wave propagation in orthotropic unidirectional fiber composites and cross ply composites.

\section{ALGORITHMS FOR CALCULATING DISPERSION CURVES}

This section includes a brief description of the methods that are used for calculating dispersion curves in FRP composites.

\subsection{Transfer Matrix Method (TMM)}

Transfer matrix method (TMM) developed by Thomson $^{19}$ and modified by Haskell ${ }^{20}$ is a reliable technique for wave propagation analysis in layered media; its advantage is that it condenses the multi-layered system into four equations (for the case of decoupled SH waves) or six equations relating the boundary conditions at the first and the last interfaces. It eliminates all other intermediate interfaces, which save a lot of computational time and complexity. Hence, TMM is favorable. One drawback TMM suffers is its numerical instability of the solution at large frequency-thickness products ${ }^{21}$. TMM is well covered by Nayfeh ${ }^{15}$.

\subsection{Global Matrix Method (GMM)}

Global matrix was first developed by Knopoff ${ }^{22}$. It combines stresses and displacements at the boundaries of each layer with boundary conditions and assembles them in one single matrix. Compared to Thomson-Haskell ${ }^{19},{ }^{20}$ transfer matrix technique, global matrix has the advantage that it remains stable at high frequency-thickness products. GMM shows the same base matrix whether for real or imaginary wave numbers, vacuum, liquid, or solid half-space ${ }^{21}$. The disadvantage is that the global matrix ends up as a large matrix for laminates with large numbers of layers.

\subsection{Semi Analytical Finite Element (SAFE)}

SAFE discretizes the structure cross section allowing different cross sections to be analyzed, because of the finite element discretizing in cross section. At the same time it solves analytically for wave propagation direction; which makes it more efficient in terms of computational time and memory than a complete FEM $^{23},{ }^{24}$. The advantage of discretizing the cross section is that it allows the modeling of any arbitrary cross sections (e.g. rail ${ }^{25},{ }^{26}$ ). Also, in general the material is defined in FEM by stiffness matrix; that makes SAFE method very straight forward for application of anisotropic materials. SAFE is becoming popular for analyzing guided wave propagation in composites ${ }^{26}$.

\subsection{Local Interaction Simulation Approach (LISA)}

LISA discretizes the system into a lattice like finite difference method and its formulation is based on elastodynamic equations. The advantage of LISA appears when discontinuities or changes need to be applied to the material properties; those changes are treated by simply modifying the properties of the lattice at the corresponding locations. LISA is well studied by Delsanto ${ }^{27}$ for 3-D case. A well-organized formulation was reported in $\operatorname{Ref}^{28}$ with experimental validations of LISA for both isotropic and anisotropic media. Another study ${ }^{29}$ compares LISA approach with experimental results using laser vibrometer for elastic plates.

\subsection{Equivalent Matrix Method (EMM)}

EMM is a very quick and reliable approach for analyzing cross ply laminates due to the fact that transformation matrix between 0 and 90 degrees is straight forward and can be done manually It can be applied for generally orientated layers as well; though the developed code in this study is only for cross ply laminates. Fill direction is the reference fiber direction, and warp is the perpendicular direction. The corresponding warp stiffness is calculated and the average is determined, then, any of previous methods can be used. EMM is used in Ref. ${ }^{30}$. 


\section{THEORY}

In this section, a brief mathematical formulation of TMM is discussed, followed by instability of TMM, then the framework in $\operatorname{Refs}^{18,31}$ is used for eliminating instability of TMM by using SMM.

\subsection{TMM formulation}

Considering the composite plate layer is in $x_{1}, x_{2}$ plane with wave propagation along $x_{1}$ direction (Figure 1). The angle of fibers with respect to direction of wave propagation is $\theta$. The layer stiffness matrix in global coordinates is

$$
\bar{c}]=[T]^{-1}[c][T]^{-t}
$$

where $T$ is the transformation matrix and can be found from many composites textbooks, e.g. ${ }^{32}$. And $c$ is the layer stiffness matrix in local coordinates. The maximum anisotropy we are considering is orthotropic layer; once the layer is rotated by angle $\theta$, it becomes monoclinic anisotropy. Equation of motion is

$$
\left\{\begin{array}{l}
\nabla \cdot\left(\mathbf{c}: \nabla_{s} \mathbf{u}\right)=\rho \frac{\partial^{2} \mathbf{u}}{\partial t^{2}} \\
\nabla_{s} \mathbf{u}=\mathbf{S}
\end{array}\right.
$$

where $\rho$ is the density, $\mathbf{S}$ is the strains tensor, $\mathbf{u}$ is the displacements vector and is decomposed into the three axes as

$$
\left(u_{1}, u_{2}, u_{3}\right)=\left(U_{1}, U_{2}, U_{3}\right) e^{i \xi\left(x_{1}+\alpha x_{3}-v t\right)}
$$

where $\xi$ is the wave number in the $x_{1}$ direction, $v=\omega / \xi$ is the phase velocity, $\omega$ is the angular frequency, $\alpha$ is the ratio between wave number in the thickness direction $x_{3}$ and $x_{1}$ direction, and $U_{i}$ is the displacement amplitude. Substituting Eq. (3) into Eq. (2) and cancelling the exponential terms yields

$$
\left\{\begin{array}{l}
\left(c_{11}+c_{55} \alpha^{2}-\rho v^{2}\right) U_{1}+\left(c_{16}+c_{45} \alpha^{2}\right) U_{2}+\left(c_{13}+c_{55}\right) \alpha U_{3}=0 \\
\left(c_{16}+c_{45} \alpha^{2}\right) U_{1}+\left(c_{66}+c_{44} \alpha^{2}-\rho v^{2}\right) U_{2}+\left(c_{45}+c_{36}\right) \alpha U_{3}=0 \\
\left(c_{13}+c_{55}\right) \alpha U_{1}+\left(c_{36}+c_{45}\right) \alpha U_{2}+\left(c_{55}+c_{33} \alpha^{2}-\rho v^{2}\right) U_{3}=0
\end{array}\right.
$$

This is an eigenvalue problem, and the determinant of Eq. (4) can be expressed as

$$
\alpha^{6}+A_{1} \alpha^{4}+A_{2} \alpha^{2}+A_{3}=0
$$

where $A_{i}$ values are found in $\operatorname{Ref}^{15}$.
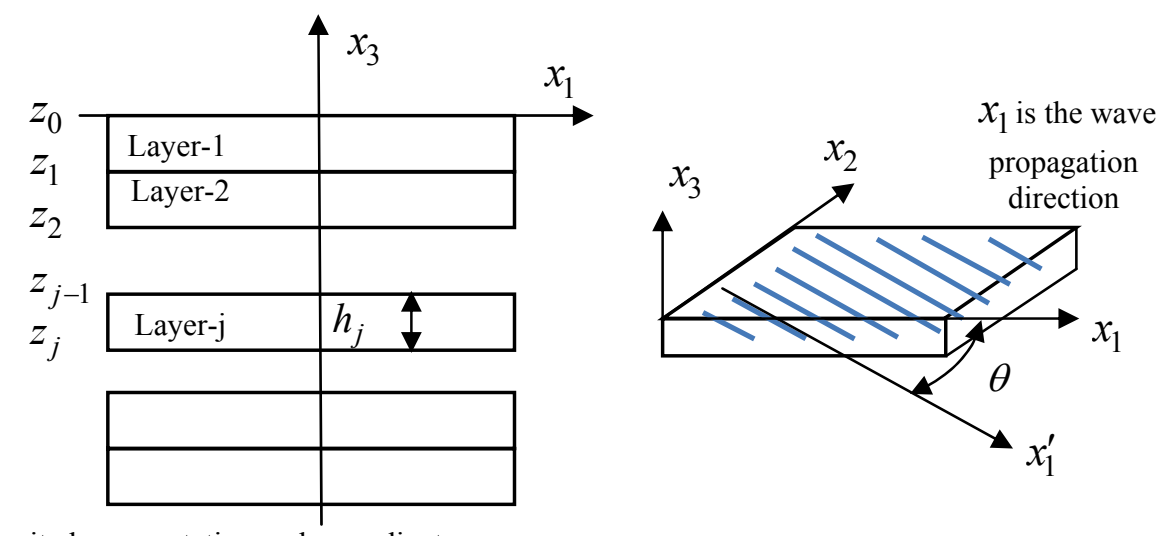

Figure 1. Composite layers notation and co-ordinates

By solving Eq. (5) symbolically; it can be shown that the eigenvalues $\alpha_{i}$ are in pairs, i.e.

$$
\alpha_{2}=-\alpha_{1}, \alpha_{4}=-\alpha_{3}, \alpha_{6}=-\alpha_{5}
$$


Using any two equations in Eq.(4), we find the displacements ratios (i.e. eigenvectors). However, careful selection of the two equations is important. If this algorithm is used for isotropic metallic layer or a composite layer that is almost isotropic, the displacements ratio $W_{q}=U_{3 q} / U_{1 q}$ suffers a singularity situation. Therefore, the ratios documented in ${ }^{15,16}$ are exchanged by ${ }^{18}$

$$
\left\{\begin{array}{l}
V_{q}=\frac{U_{2 q}}{U_{1 q}}=\frac{\left(c_{11}+c_{55} \alpha_{q}^{2}-\rho v^{2}\right)\left(c_{45}+c_{36}\right) \alpha_{q}-\left(c_{16}+c_{45} \alpha_{q}^{2}\right)\left(c_{13}+c_{55}\right) \alpha_{q}}{\left(c_{13}+c_{55}\right) \alpha_{q}\left(c_{66}+c_{44} \alpha_{q}^{2}-\rho v^{2}\right)-\left(c_{16}+c_{45} \alpha_{q}^{2}\right)\left(c_{45}+c_{36}\right) \alpha_{q}} \\
W_{q}=\frac{U_{3 q}}{U_{1 q}}=\frac{\left(c_{11}+c_{55} \alpha_{q}^{2}-\rho v^{2}\right)\left(c_{66}+c_{44} \alpha_{q}^{2}-\rho v^{2}\right)-\left(c_{16}+c_{45} \alpha_{q}^{2}\right)^{2}}{\left(c_{16}+c_{45} \alpha_{q}^{2}\right)\left(c_{45}+c_{36}\right) \alpha_{q}-\left(c_{13}+c_{55}\right) \alpha_{q}\left(c_{66}+c_{44} \alpha_{q}^{2}-\rho v^{2}\right)}
\end{array}\right.
$$

where $q$ represents the solution number from one to six. The displacements in Eq. (3) are written as

$$
\left(u_{1}, u_{2}, u_{3}\right)=\sum_{q=1}^{6}\left(1, V_{q}, W_{q}\right) U_{1 q} e^{i \xi\left(x_{1}+\alpha_{q} x_{3}-v t\right)}
$$

The stresses are

$$
\left\{\begin{array}{c}
T_{1} \\
T_{2} \\
T_{3} \\
T_{4} \\
T_{5} \\
T_{6}
\end{array}\right\}=i \xi \sum_{q}\left\{\begin{array}{c}
c_{11}+\alpha_{q} c_{13} W_{q}+c_{16} V_{q} \\
c_{12}+\alpha_{q} c_{23} W_{q}+c_{26} V_{q} \\
c_{13}+\alpha_{q} c_{33} W_{q}+c_{36} V_{q} \\
\alpha_{q} c_{44} V_{q}+c_{45}\left(\alpha_{q}+W_{q}\right) \\
\alpha_{q} c_{45} V_{q}+c_{55}\left(\alpha_{q}+W_{q}\right) \\
c_{16}+\alpha_{q} c_{36} W_{q}+c_{66} V_{q}
\end{array}\right\} U_{1 q} e^{i \xi\left(x_{1}+\alpha_{q} x_{3}-v t\right)}
$$

The stresses that are of interest are $\sigma_{33}, \sigma_{13}, \sigma_{23}$. Stress-free boundary condition is applied on them, define

$$
\left(\sigma_{33}^{*}, \sigma_{13}^{*}, \sigma_{23}^{*}\right)=\left(T_{3}^{*}, T_{5}^{*}, T_{4}^{*}\right)=\sum_{q=1}^{6}\left(d_{1 q}, d_{2 q}, d_{3 q}\right) U_{1 q} e^{i \xi\left(x_{1}+\alpha_{q} x_{3}-v t\right)}
$$

Where $\sigma^{*}=\sigma / i \xi$, and $d_{1 q}, d_{2 q}, d_{3 q}$ are contracted terms from Eq. (9).

The relation for wave propagation in a layer with combining displacement and stress relations is

$$
\left[\begin{array}{c}
u_{1} \\
u_{2} \\
u_{3} \\
\sigma_{33}^{*} \\
\sigma_{13}^{*} \\
\sigma_{23}^{*}
\end{array}\right]=\left[\begin{array}{cccccc}
1 & 1 & 1 & 1 & 1 & 1 \\
V_{1} & V_{1} & V_{3} & V_{3} & V_{5} & V_{5} \\
W_{1} & -W_{1} & W_{3} & -W_{3} & W_{5} & -W_{5} \\
d_{11} & d_{11} & d_{13} & d_{13} & d_{15} & d_{15} \\
d_{21} & -d_{21} & d_{23} & -d_{23} & d_{25} & -d_{25} \\
d_{31} & -d_{31} & d_{31} & -d_{31} & d_{35} & -d_{35}
\end{array}\right]\left[\begin{array}{c}
U_{11} e^{i \xi \alpha_{1} x_{3}} \\
U_{11} e^{-i \xi \alpha_{1} x_{3}} \\
U_{13} e^{i \xi \alpha_{3} x_{3}} \\
U_{13} e^{-i \xi \alpha_{3} x_{3}} \\
U_{15} e^{i \xi \alpha_{5} x_{3}} \\
U_{15} e^{-i \xi \alpha_{5} x_{3}}
\end{array}\right] e^{i \xi\left(x_{1}-v t\right)}
$$

The idea of TMM is relating the layer properties and boundary conditions at the top and bottom surfaces with other layers. This is done by applying continuity of displacements and equilibrium of stresses. The layer transfer matrix $A_{k}$ relates the displacements and stresses of the bottom of the layer to those of the top of the layer

$$
\left\{\begin{array}{l}
\left\{u^{\text {Bottom }}\right\} \\
\left\{\sigma^{\text {Bottom }}\right\}
\end{array}\right\}=\left[\begin{array}{ll}
{\left[A_{u u}\right]} & {\left[A_{u \sigma}\right]} \\
{\left[A_{u \sigma}\right]} & {\left[A_{\sigma \sigma}\right]}
\end{array}\right]\left\{\begin{array}{l}
\left\{u^{T o p}\right\} \\
\left\{\sigma^{T o p}\right\}
\end{array}\right\}
$$

where $A_{k}$ is the $4 \times 4$ matrix of Eq. (12). Call the $6 \times 6$ matrix of Eq. (11) $X$, the vector of $U_{1 q}$ elements $U$, and the diagonal matrix of elements $e^{i \xi \alpha_{q} x_{3}} H$. Hence,

$$
A_{k}=X_{k} H_{k} X_{k}^{-1}
$$


The total TM is calculated by multiplying the transfer matrix of individual layers consecutively. And to satisfy stress free boundary condition for the whole laminate, $\sigma^{T o p}$ and $\sigma^{\text {Bottom }}$ in Eq. (12) are set to zero, hence the characteristic equation to find dispersion phase velocities versus wavenumbers is

$$
\left|A_{u \sigma}\right|=0
$$

Usually Eq. (14) is solved numerically to find dispersion phase velocities versus wavenumbers or frequencies.

\subsection{Instability of Transfer Matrix Method}

Rokhlin $^{18}$ showed that due to refraction within one or more layers of the laminate, some of the plane waves can be internally reflected, meaning that their partial waves will be evanescent within the layer, i.e. propagating constant will be imaginary in the exponential $e^{ \pm i \xi x_{3}}$. Depending on the thickness and the frequency the real term $e^{\xi x_{3}}$ can be very large or very small. The TM formulation in itself has no deficiency. But numerical computation of this real exponential that rises and falls quickly suffers instabilities frequently (Figure 2).

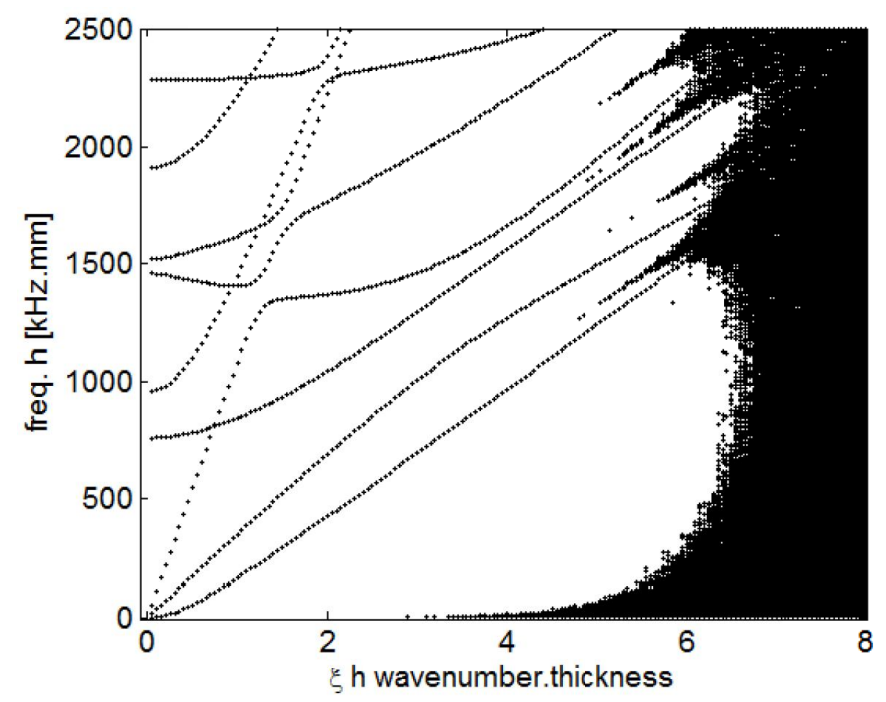

Figure 2. Instability of TMM for unidirectional carbon fiber lamina with $45^{\circ}$ fibers

\subsection{Stiffness Matrix Method}

There have been many publications proposing reformulation of equations to avoid this problem e.g. ${ }^{31}$. The method is based on using stiffness matrix method (SMM) instead of TMM; this is done by re-arranging terms of the TMM such that displacements at both the top and the bottom of the $\mathrm{j}$ layer are in a single column matrix. Similarly, tractions at both the top and the bottom of the $\mathrm{j}$ layer are combined in one single column matrix. The transfer function between them will be the SMM or $\mathbf{K}^{\mathbf{j}}$,

$$
\left[\begin{array}{c}
\sigma_{j-1} \\
\sigma_{j}
\end{array}\right]=\mathbf{K}^{\mathbf{j}}\left[\begin{array}{c}
u_{j-1} \\
u_{j}
\end{array}\right]
$$

This is in contrast to the transfer matrix in which displacement and traction for each boundary are combined in the one single column vector, Eq. (12). The recursive approach to find the "total" stiffness matrix of all layers is well documented in the Ref ${ }^{18}$ That approach is not as straight forward as the one applied for finding the total TM. For total $\mathrm{TM}$, we simply multiply transfer matrix of all individual layers. The total stiffness matrix for two layers is ${ }^{18}$

$$
\left[\begin{array}{c}
\sigma_{0} \\
\sigma_{2}
\end{array}\right]=\left[\begin{array}{cc}
K_{11}^{A}+K_{12}^{A}\left(K_{11}^{B}-K_{22}^{A}\right)^{-1} K_{21}^{A} & -K_{12}^{A}\left(K_{11}^{B}-K_{22}^{A}\right)^{-1} K_{12}^{B} \\
K_{21}^{B}\left(K_{11}^{B}-K_{22}^{A}\right)^{-1} K_{21}^{A} & K_{22}^{B}-K_{21}^{B}\left(K_{11}^{B}-K_{22}^{A}\right)^{-1} K_{12}^{B}
\end{array}\right]\left[\begin{array}{l}
u_{0} \\
u_{2}
\end{array}\right]
$$


For complete understanding and ease of applying this approach, we show the detailed closed-form equations to be used and flow chart of this framework (Figure 3).

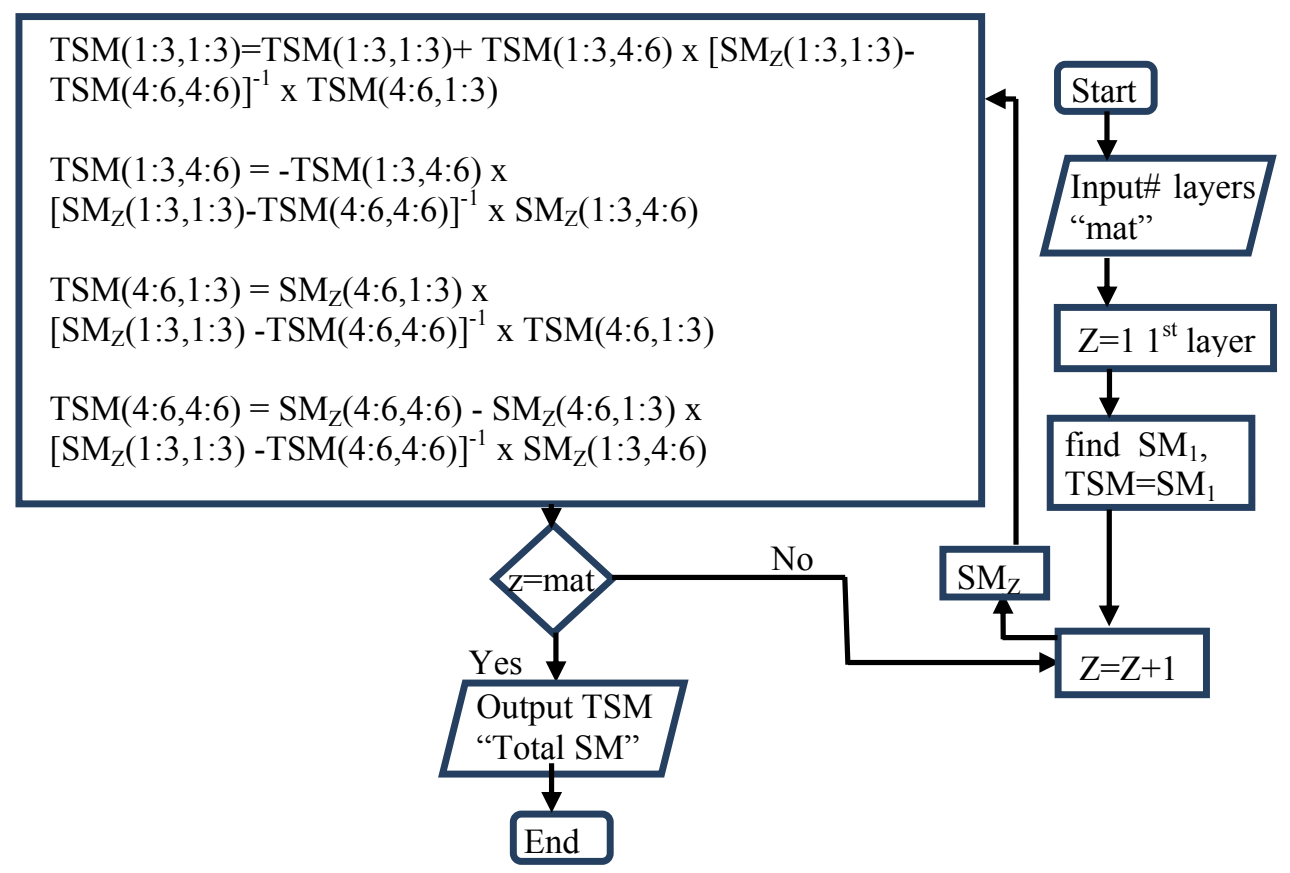

Figure 3 Flow chart of total SM recursive algorithm

Recall Eq.(11), and that the diagonal matrix of elements $e^{i \xi \alpha_{q} x_{3}}$ is indicated by $H$, the way the equations are arranged in the SMM needs to consider removing $H$ values from critical locations (at one column or the diagonal) as ${ }^{18}$,

$$
\left[\begin{array}{c}
\sigma_{j-1} \\
\sigma_{j}
\end{array}\right]=\left[\begin{array}{cc}
D^{-} & D^{+} H^{+} \\
D^{-} H^{-} & D^{+}
\end{array}\right]_{j}\left[\begin{array}{cc}
P^{-} & P^{+} H^{+} \\
P^{-} H^{-} & P^{+}
\end{array}\right]_{j}^{-1}\left[\begin{array}{c}
u_{j-1} \\
u_{j}
\end{array}\right]
$$

where $P_{\mathrm{s}}$ represent the coefficients associated with displacements, i.e. $1_{\mathrm{s}}$ and $W_{3}, W_{5}$ of equation (11), while $D_{\mathrm{s}}$ are the $d_{13}, d_{15} \ldots d_{25}$ associated with stresses. Our objective here is to formulate the equations on the form of $6 \times 6$ matrix on the form which Rokhlin recommended, so it is easy to be coded, instead of writing the matrices in compact forms. So in the following pages, we make the analytical development needed to get the $4 \times 4 \mathrm{SM}$ form and show that $H$ values are no longer on diagonal or single column. In the following pages, analytical development is shown to get the ( $6 \times 6) \mathrm{SM}$ form. The way this is done is by factoring out other exponentials that are going to be merged with the displacement amplitudes of the partial waves, i.e. $U_{\mathrm{lq}}$. By doing that, we eliminate the growing nature of the exponentials as the number of layers increase, or as the wavenumber-thickness increases.

Expanding the system of equations of (11) yields,

$$
\begin{aligned}
& u_{1}=U_{11} e^{i \xi \alpha_{1} x_{3}}+U_{11} e^{-i \xi \alpha_{1} x_{3}}+U_{13} e^{i \xi \alpha_{3} x_{3}}+U_{13} e^{-i \xi \alpha_{3} x_{3}}+U_{15} e^{i \xi \alpha_{5} x_{3}}+U_{15} e^{-i \xi \alpha_{5} x_{3}} \\
& u_{2}=U_{11} V_{1} e^{i \xi \alpha_{1} x_{3}}+U_{11} V_{1} e^{-i \xi \alpha_{1} x_{3}}+U_{13} V_{3} e^{i \xi \alpha_{3} x_{3}}+U_{13} V_{3} e^{-i \xi \alpha_{3} x_{3}}+U_{15} V_{5} e^{i \xi \alpha_{5} x_{3}}+U_{15} V_{5} e^{-i \xi \alpha_{5} x_{3}} \\
& u_{3}=U_{11} W_{1} e^{i \xi \alpha_{1} x_{3}}-U_{11} W_{1} e^{-i \xi \alpha_{1} x_{3}}+U_{13} W_{3} e^{i \xi \alpha_{3} x_{3}}-U_{13} W_{3} e^{-i \xi \alpha_{3} x_{3}}+U_{15} W_{5} e^{i \xi \alpha_{5} x_{3}}-U_{15} W_{5} e^{-i \xi \alpha_{5} x_{3}} \\
& \sigma_{33}^{*}=U_{11} d_{11} e^{i \xi \alpha_{1} x_{3}}+U_{11} d_{11} e^{-i \xi \alpha_{1} x_{3}}+U_{13} d_{13} e^{i \xi \alpha_{3} x_{3}}+U_{13} d_{13} e^{-i \xi \alpha_{3} x_{3}}+U_{15} d_{15} e^{i \xi \alpha_{5} x_{3}}+U_{15} d_{15} e^{-i \xi \alpha_{5} x_{3}} \\
& \sigma_{13}^{*}=U_{11} d_{21} e^{i \xi \alpha_{1} x_{3}}-U_{11} d_{21} e^{-i \xi \alpha_{1} x_{3}}+U_{13} d_{23} e^{i \xi \alpha_{3} x_{3}}-U_{13} d_{23} e^{-i \xi \alpha_{3} x_{3}}+U_{15} d_{25} e^{i \xi \alpha_{5} x_{3}}-U_{15} d_{25} e^{-i \xi \alpha_{5} x_{3}} \\
& \sigma_{23}^{*}=U_{11} d_{31} e^{i \xi \alpha_{1} x_{3}}-U_{11} d_{31} e^{-i \xi \alpha_{1} x_{3}}+U_{13} d_{33} e^{i \xi \alpha_{3} x_{3}}-U_{13} d_{33} e^{-i \xi \alpha_{3} x_{3}}+U_{15} d_{35} e^{i \xi \alpha_{5} x_{3}}-U_{15} d_{35} e^{-i \xi \alpha_{5} x_{3}}
\end{aligned}
$$


Rearranging yields,

$$
\begin{aligned}
& u_{1}=\left(U_{11} e^{i \xi \alpha_{1} z_{j-1}}\right) e^{i \xi \alpha_{1}\left(x_{3}-z_{j-1}\right)}+\left(U_{11} e^{-i \xi \alpha_{1} z_{j}}\right) e^{-i \xi \alpha_{1}\left(x_{3}-z_{j}\right)}+\left(U_{13} e^{i \xi \alpha_{3} z_{j-1}}\right) e^{i \xi \alpha_{3}\left(x_{3}-z_{j-1}\right)} \\
& +\left(U_{13} e^{-i \xi \alpha_{3} z_{j}}\right) e^{-i \xi \alpha_{3}\left(x_{3}-z_{j}\right)}+\left(U_{15} e^{i \xi \alpha_{5} z_{j-1}}\right) e^{i \xi \alpha_{5}\left(x_{3}-z_{j-1}\right)}+\left(U_{15} e^{-i \xi \alpha_{5} z_{j}}\right) e^{-i \xi \alpha_{5}\left(x_{3}-z_{j}\right)} \\
& u_{2}=\left(U_{11} e^{i \xi \alpha_{1} z_{j-1}}\right) V_{1} e^{i \xi \alpha_{1}\left(x_{3}-z_{j-1}\right)}+\left(U_{11} e^{-i \xi \alpha_{1} z_{j}}\right) V_{1} e^{-i \xi \alpha_{1}\left(x_{3}-z_{j}\right)}+\left(U_{13} e^{i \xi \alpha_{3} z_{j-1}}\right) V_{3} e^{i \xi \alpha_{3}\left(x_{3}-z_{j-1}\right)} \\
& +\left(U_{13} e^{-i \xi \alpha_{3} z_{j}}\right) V_{3} e^{-i \xi \alpha_{3}\left(x_{3}-z_{j}\right)}+\left(U_{15} e^{i \xi \alpha_{5} z_{j-1}}\right) V_{5} e^{i \xi \alpha_{5}\left(x_{3}-z_{j-1}\right)}+\left(U_{15} e^{-i \xi \alpha_{5} z_{j}}\right) V_{5} e^{-i \xi \alpha_{5}\left(x_{3}-z_{j}\right)} \\
& u_{3}=\left(U_{11} e^{i \xi \alpha_{1} z_{j-1}}\right) W_{1} e^{i \xi \alpha_{1}\left(x_{3}-z_{j-1}\right)}-\left(U_{11} e^{-i \xi \alpha_{1} z_{j}}\right) W_{1} e^{-i \xi \alpha_{1}\left(x_{3}-z_{j}\right)}+\left(U_{13} e^{i \xi \alpha_{3} z_{j-1}}\right) W_{3} e^{i \xi \alpha_{3}\left(x_{3}-z_{j-1}\right)} \\
& -\left(U_{13} e^{-i \xi \alpha_{3} z_{j}}\right) W_{3} e^{-i \xi \alpha_{3}\left(x_{3}-z_{j}\right)}+\left(U_{15} e^{i \xi \alpha_{5} z_{j-1}}\right) W_{5} e^{i \xi \alpha_{5}\left(x_{3}-z_{j-1}\right)}-\left(U_{15} e^{-i \xi \alpha_{5} z_{j}}\right) W_{5} e^{-i \xi \alpha_{5}\left(x_{3}-z_{j}\right)} \\
& \sigma_{33}^{*}=\left(U_{11} e^{i \xi \alpha_{1} z_{j-1}}\right) d_{11} e^{i \xi \alpha_{1}\left(x_{3}-z_{j-1}\right)}+\left(U_{11} e^{-i \xi \alpha_{1} z_{j}}\right) d_{11} e^{-i \xi \alpha_{1}\left(x_{3}-z_{j}\right)}+\left(U_{13} e^{i \xi \alpha_{3} z_{j-1}}\right) d_{13} e^{i \xi \alpha_{3}\left(x_{3}-z_{j-1}\right)} \\
& +\left(U_{13} e^{-i \xi \alpha_{3} z_{j}}\right) d_{13} e^{-i \xi \alpha_{3}\left(x_{3}-z_{j}\right)}+\left(U_{15} e^{i \xi \alpha_{5} z_{j-1}}\right) d_{15} e^{i \xi \alpha_{5}\left(x_{3}-z_{j-1}\right)}+\left(U_{15} e^{-i \xi \alpha_{5} z_{j}}\right) d_{15} e^{-i \xi \alpha_{5}\left(x_{3}-z_{j}\right)} \\
& \sigma_{13}^{*}=\left(U_{11} e^{i \xi \alpha_{1} z_{j-1}}\right) d_{21} e^{i \xi \alpha_{1}\left(x_{3}-z_{j-1}\right)}-\left(U_{11} e^{-i \xi \alpha_{1} z_{j}}\right) d_{21} e^{-i \xi \alpha_{1}\left(x_{3}-z_{j}\right)}+\left(U_{13} e^{i \xi \alpha_{3} z_{j-1}}\right) d_{23} e^{i \xi \alpha_{3}\left(x_{3}-z_{j-1}\right)} \\
& -\left(U_{13} e^{-i \xi \alpha_{3} z_{j}}\right) d_{23} e^{-i \xi \alpha_{3}\left(x_{3}-z_{j}\right)}+\left(U_{15} e^{i \xi \alpha_{5} z_{j-1}}\right) d_{25} e^{i \xi \alpha_{5}\left(x_{3}-z_{j-1}\right)}-\left(U_{15} e^{-i \xi \alpha_{5} z_{j}}\right) d_{25} e^{-i \xi \alpha_{5}\left(x_{3}-z_{j}\right)} \\
& \sigma_{23}^{*}=\left(U_{11} e^{i \xi \alpha_{1} z_{j-1}}\right) d_{31} e^{i \xi \alpha_{1}\left(x_{3}-z_{j-1}\right)}-\left(U_{11} e^{-i \xi \alpha_{1} z_{j}}\right) d_{31} e^{-i \xi \alpha_{1}\left(x_{3}-z_{j}\right)}+\left(U_{13} e^{i \xi \alpha_{3} z_{j-1}}\right) d_{33} e^{i \xi \alpha_{3}\left(x_{3}-z_{j-1}\right)} \\
& -\left(U_{13} e^{-i \xi \alpha_{3} z_{j}}\right) d_{33} e^{-i \xi \alpha_{3}\left(x_{3}-z_{j}\right)}+\left(U_{15} e^{i \xi \alpha_{5} z_{j-1}}\right) d_{35} e^{i \xi \alpha_{5}\left(x_{3}-z_{j-1}\right)}-\left(U_{15} e^{-i \xi \alpha_{5} z_{j}}\right) d_{35} e^{-i \xi \alpha_{5}\left(x_{3}-z_{j}\right)}
\end{aligned}
$$

Rearranging displacements and stresses of equation (19) in a matrix form.

For the top surface " $\mathrm{j}-1$ " of the layer, i.e. $x_{3}=z_{j-1}$, we get,

$$
\left[\begin{array}{l}
u_{1}^{j-1} \\
u_{2}^{j-1} \\
u_{3}^{j-1} \\
\sigma_{33}^{* j-1} \\
\sigma_{13}^{j-1} \\
\sigma_{23}^{* j-1}
\end{array}\right]=\left[\begin{array}{cccccc}
1 & e^{i \xi \alpha_{1} h_{j}} & 1 & e^{i \xi \alpha_{3} h_{j}} & 1 & e^{i \xi \alpha_{5} h_{j}} \\
V_{1} & V_{1} e^{i \xi \alpha_{1} h_{j}} & V_{3} & V_{3} e^{i \xi \alpha_{3} h_{j}} & V_{5} & V_{5} e^{i \xi \alpha_{5} h_{j}} \\
W_{1} & -W_{1} e^{i \xi \alpha_{1} h_{j}} & W_{3} & -W_{3} e^{i \xi \alpha_{3} h_{j}} & W_{5} & -W_{5} e^{i \xi \alpha_{5} h_{j}} \\
d_{11} & d_{11} e^{i \xi \alpha_{1} h_{j}} & d_{13} & d_{13} e^{i \xi \alpha_{3} h_{j}} & d_{15} & d_{15} e^{i \xi \alpha_{5} h_{j}} \\
d_{21} & -d_{21} e^{i \xi \alpha_{1} h_{j}} & d_{23} & -d_{23} e^{i \xi \alpha_{3} h_{j}} & d_{25} & -d_{25} e^{i \xi \alpha_{5} h_{j}} \\
d_{31} & -d_{31} e^{i \xi \alpha_{1} h_{j}} & d_{33} & -d_{33} e^{i \xi \alpha_{3} h_{j}} & d_{35} & -d_{35} e^{i \xi \alpha_{5} h_{j}}
\end{array}\right]\left[\begin{array}{l}
U_{11} e^{i \xi \alpha_{1} z_{j-1}} \\
U_{11} e^{-i \xi \alpha_{1} z_{j}} \\
U_{13} e^{i \xi \alpha_{3} z_{j-1}} \\
U_{13} e^{-i \xi \alpha_{3} z_{j}} \\
U_{15} e^{i \xi \alpha_{5} z_{j-1}} \\
U_{15} e^{-i \xi \alpha_{5} z_{j}}
\end{array}\right]
$$

And rearranging for the bottom surface " $\mathrm{j}$ ", i.e. $x_{3}=z_{j}$ yields,

$$
\left[\begin{array}{c}
u_{1}^{j} \\
u_{2}^{j} \\
u_{3}^{j} \\
\sigma_{33}^{* j} \\
\sigma_{13}^{* j} \\
\sigma_{23}^{* j}
\end{array}\right]=\left[\begin{array}{cccccc}
e^{i \xi \alpha_{1} h_{j}} & 1 & e^{i \xi \alpha_{3} h_{j}} & 1 & e^{i \xi \alpha_{5} h_{j}} & 1 \\
V_{1} e^{i \xi \alpha_{1} h_{j}} & V_{1} & V_{3} e^{i \xi \alpha_{3} h_{j}} & V_{3} & V_{5} e^{i \xi \alpha_{5} h_{j}} & V_{5} \\
W_{1} e^{i \xi \alpha_{1} h_{j}} & -W_{1} & W_{3} e^{i \xi \alpha_{3} h_{j}} & -W_{3} & W_{5} e^{i \xi \alpha_{5} h_{j}} & -W_{5} \\
d_{11} e^{i \xi \alpha_{1} h_{j}} & d_{11} & d_{13} e^{i \xi \alpha_{3} h_{j}} & d_{13} & d_{15} e^{i \xi \alpha_{5} h_{j}} & d_{15} \\
d_{21} e^{i \xi \alpha_{1} h_{j}} & -d_{21} & d_{23} e^{i \xi \alpha_{3} h_{j}} & -d_{23} & d_{25} e^{i \xi \alpha_{5} h_{j}} & -d_{25} \\
d_{31} e^{i \xi \alpha_{1} h_{j}} & -d_{31} & d_{33} e^{i \xi \alpha_{3} h_{j}} & -d_{33} & d_{35} e^{i \xi \alpha_{5} h_{j}} & -d_{35}
\end{array}\right]\left[\begin{array}{l}
U_{11} e^{i \xi \alpha_{1} z_{j-1}} \\
U_{11} e^{-i \xi \alpha_{1} z_{j}} \\
U_{13} e^{i \xi \alpha_{3} z_{j-1}} \\
U_{13} e^{-i \xi \alpha_{3} z_{j}} \\
U_{15} e^{i \xi \alpha_{5} z_{j-1}} \\
U_{15} e^{-i \xi \alpha_{5} z_{j}}
\end{array}\right]
$$


The right hand side array $\{\mathbf{U}\}$ of modal displacements is always the same and will be eliminated later. Also Eqs. (20), (21) are rearranged such that we keep exponentials containing the layer thickness at one side of the matrix. This is done by rearranging the elements of $\{\mathbf{U}\}$ array, without changing the rows sequence of left hand side arrays, equation (20) is simplified as

$$
\left[\begin{array}{l}
u_{1}^{j-1} \\
u_{2}^{j-1} \\
u_{3}^{j-1} \\
\sigma_{33}^{* j-1} \\
\sigma_{13}^{j-1} \\
\sigma_{23}^{j-1}
\end{array}\right]=\left[\begin{array}{cccccc}
1 & 1 & 1 & e^{i \xi \alpha_{1} h_{j}} & e^{i \xi \alpha_{3} h_{j}} & e^{i \xi \alpha_{5} h_{j}} \\
V_{1} & V_{3} & V_{5} & V_{1} e^{i \xi \alpha_{1} h_{j}} & V_{3} e^{i \xi \alpha_{3} h_{j}} & V_{5} e^{i \xi \alpha_{5} h_{j}} \\
W_{1} & W_{3} & W_{5} & -W_{1} e^{i \xi \alpha_{1} h_{j}} & -W_{3} e^{i \xi \alpha_{3} h_{j}} & -W_{5} e^{i \xi \alpha_{5} h_{j}} \\
d_{11} & d_{13} & d_{15} & d_{11} e^{i \xi \alpha_{1} h_{j}} & d_{13} e^{i \xi \alpha_{3} h_{j}} & d_{15} e^{i \xi \alpha_{5} h_{j}} \\
d_{21} & d_{23} & d_{25} & -d_{21} e^{i \xi \alpha_{1} h_{j}} & -d_{23} e^{i \xi \alpha_{3} h_{j}} & -d_{25} e^{i \xi \alpha_{5} h_{j}} \\
d_{31} & d_{33} & d_{35} & -d_{31} e^{i \xi \alpha_{1} h_{j}} & -d_{33} e^{i \xi \alpha_{3} h_{j}} & -d_{35} e^{i \xi \alpha_{5} h_{j}}
\end{array}\right]\left[\begin{array}{l}
U_{11} e^{i \xi \alpha_{1} z_{j-1}} \\
U_{13} e^{i \xi \alpha_{3} z_{j-1}} \\
U_{15} e^{i \xi \alpha_{5} z_{j-1}} \\
U_{11} e^{-i \xi \alpha_{1} z_{j}} \\
U_{13} e^{-i \xi \alpha_{3} z_{j}} \\
U_{15} e^{-i \xi \alpha_{5} z_{j}}
\end{array}\right]
$$

And Eq. (21) is simplified as,

$$
\left[\begin{array}{c}
u_{1}^{j} \\
u_{2}^{j} \\
u_{3}^{j} \\
\sigma_{33}^{* j} \\
\sigma_{13}^{* j} \\
\sigma_{23}^{* j}
\end{array}\right]=\left[\begin{array}{cccccc}
e^{i \xi \alpha_{1} h_{j}} & e^{i \xi \alpha_{3} h_{j}} & e^{i \xi \alpha_{5} h_{j}} & 1 & 1 & 1 \\
V_{1} e^{i \xi \alpha_{1} h_{j}} & V_{3} e^{i \xi \alpha_{3} h_{j}} & V_{5} e^{i \xi \alpha_{5} h_{j}} & V_{1} & V_{3} & V_{5} \\
W_{1} e^{i \xi \alpha_{1} h_{j}} & W_{3} e^{i \xi \alpha_{3} h_{j}} & W_{5} e^{i \xi \alpha_{5} h_{j}} & -W_{1} & -W_{3} & -W_{5} \\
d_{11} e^{i \xi \alpha_{1} h_{j}} & d_{13} e^{i \xi \alpha_{3} h_{j}} & d_{15} e^{i \xi \alpha_{5} h_{j}} & d_{11} & d_{13} & d_{15} \\
d_{21} e^{i \xi \alpha_{1} h_{j}} & d_{23} e^{i \xi \alpha_{3} h_{j}} & d_{25} e^{i \xi \alpha_{5} h_{j}} & -d_{21} & -d_{23} & -d_{25} \\
d_{31} e^{i \xi \alpha_{1} h_{j}} & d_{33} e^{i \xi \alpha_{3} h_{j}} & d_{35} e^{i \xi \alpha_{5} h_{j}} & -d_{31} & -d_{33} & -d_{35}
\end{array}\right]\left[\begin{array}{l}
U_{11} e^{i \xi \alpha_{1} z_{j-1}} \\
U_{13} e^{i \xi \alpha_{3} z_{j-1}} \\
U_{15} e^{i \xi \alpha_{5} z_{j-1}} \\
U_{11} e^{-i \xi \alpha_{1} z_{j}} \\
U_{13} e^{-i \xi \alpha_{3} z_{j}} \\
U_{15} e^{-i \xi \alpha_{5} z_{j}}
\end{array}\right]
$$

As far as now, the instability sources still exist by having $H$ sub matrices, i.e. (elements with exponentials containing layer thickness $h_{j}$ ) in complete columns. However when we formulate SM by collecting all stresses in one array and all displacements in another array,

$$
\begin{aligned}
& {\left[\begin{array}{c}
\sigma_{33}^{* j-1} \\
\sigma_{13}^{* j-1} \\
\sigma_{23}^{* j-1} \\
\sigma_{33}^{* j} \\
\sigma_{13}^{* j} \\
\sigma_{23}^{* j}
\end{array}\right]=\left[\begin{array}{cccccc}
d_{11} & d_{13} & d_{15} & d_{11} e^{i \xi \alpha_{1} h_{j}} & d_{13} e^{i \xi \alpha_{3} h_{j}} & d_{15} e^{i \xi \alpha_{5} h_{j}} \\
d_{21} & d_{23} & d_{25} & -d_{21} e^{i \xi \alpha_{1} h_{j}} & -d_{23} e^{i \xi \alpha_{3} h_{j}} & -d_{25} e^{i \xi \alpha_{5} h_{j}} \\
d_{31} & d_{33} & d_{35} & -d_{31} e^{i \xi \alpha_{1} h_{j}} & -d_{33} e^{i \xi \alpha_{3} h_{j}} & -d_{35} e^{i \xi \alpha_{5} h_{j}} \\
d_{11} e^{i \xi \alpha_{1} h_{j}} & d_{13} e^{i \xi \alpha_{3} h_{j}} & d_{15} e^{i \xi \alpha_{5} h_{j}} & d_{11} & d_{13} & d_{15} \\
d_{21} e^{i \xi \alpha_{1} h_{j}} & d_{23} e^{i \xi \alpha_{3} h_{j}} & d_{25} e^{i \xi \alpha_{5} h_{j}} & -d_{21} & -d_{23} & -d_{25} \\
d_{31} e^{i \xi \alpha_{1} h_{j}} & d_{33} e^{i \xi \alpha_{3} h_{j}} & d_{35} e^{i \xi \alpha_{5} h_{j}} & -d_{31} & -d_{33} & -d_{35}
\end{array}\right] .} \\
& {\left[\begin{array}{cccccc}
1 & 1 & 1 & e^{i \xi \alpha_{1} h_{j}} & e^{i \xi \alpha_{3} h_{j}} & e^{i \xi \alpha_{5} h_{j}} \\
V_{1} & V_{3} & V_{5} & V_{1} e^{i \xi \alpha_{1} h_{j}} & V_{3} e^{i \xi \alpha_{3} h_{j}} & V_{5} e^{i \xi \alpha_{5} h_{j}} \\
W_{1} & W_{3} & W_{5} & -W_{1} e^{i \xi \alpha_{1} h_{j}} & -W_{3} e^{i \xi \alpha_{3} h_{j}} & -W_{5} e^{i \xi \alpha_{5} h_{j}} \\
e^{i \xi \alpha_{1} h_{j}} & e^{i \xi \alpha_{3} h_{j}} & e^{i \xi \alpha_{5} h_{j}} & 1 & 1 & 1 \\
V_{1} e^{i \xi \alpha_{1} h_{j}} & V_{3} e^{i \xi \alpha_{3} h_{j}} & V_{5} e^{i \xi \alpha_{5} h_{j}} & V_{1} & V_{3} & V_{5} \\
W_{1} e^{i \xi \alpha_{1} h_{j}} & W_{3} e^{i \xi \alpha_{3} h_{j}} & W_{5} e^{i \xi \alpha_{5} h_{j}} & -W_{1} & -W_{3} & -W_{5}
\end{array}\right]^{j-1}\left[\begin{array}{c}
u_{1}^{j-1} \\
u_{2}^{j-1} \\
u_{3}^{j-1} \\
u_{1}^{j} \\
u_{2}^{j} \\
u_{3}^{j}
\end{array}\right]}
\end{aligned}
$$

The product of the two $(6 \times 6)$ is the SM. Comparing to the system with Rokhlin ${ }^{18}$ formulation, the system condenses to

$$
\left[\begin{array}{c}
\boldsymbol{\sigma}^{j-1} \\
\boldsymbol{\sigma}^{j}
\end{array}\right]=\left[\begin{array}{ll} 
& H \\
H &
\end{array}\right] \cdot\left[\begin{array}{ll}
H & H \\
H &
\end{array}\right]^{-1}\left[\begin{array}{c}
\mathbf{u}^{j-1} \\
\mathbf{u}^{j}
\end{array}\right]
$$


In the next section, we exemplify this method by numerical examples of unidirectional CFRP composite lamina and cross ply CFRP composite laminate.

\section{NUMERICAL SIMULATIONS}

In this section, results are shown for dispersion curves (frequency-wavenumber, phase velocity, and group velocity curves), the material used as case study is T300/914 CFRP used in DISPERSE software manual ${ }^{33}$ and other studies ${ }^{26},{ }^{16}$. These material properties are used for unidirectional case studies as well as cross ply cases. GUIGUW computer package which is used in $^{26}$ was used also to verify results. The core code of GUIGUW is used with help from the developers instead of the online interface.

The layer density is $\rho=1560 \mathrm{~kg} / \mathrm{m}^{3}$, and the unidirectional layer stiffness matrix values are the values of

$$
C_{u n i}=\left(\begin{array}{cccccc}
143.8 & 6.2 & 6.2 & & & \\
6.2 & 13.3 & 6.5 & & & \\
6.2 & 6.5 & 13.3 & & & \\
& & & 3.6 & & \\
& & & & 5.7 & \\
& & & & & 5.7
\end{array}\right) G P a
$$

\subsection{Unidirectional fiber composite U0}

For orthotropic unidirectional fiber composite layer, the instability of TMM solution is shown in Figure 5a. The TMM gives a good and accurate solution in the region of $\xi h$ less than $\approx 7$. An exponential function is used to separate the frequency- wavenumber domain into two regions. The function used is $y=6.2\left(e^{1.1 x}-1\right)$, where $y$ is the frequency axis, and $x$ is the wavenumber axis. The SMM solution alone showed a stable solution in the region $\xi h>7$. However, it did not give a correct solution pattern at the low wavenumber region

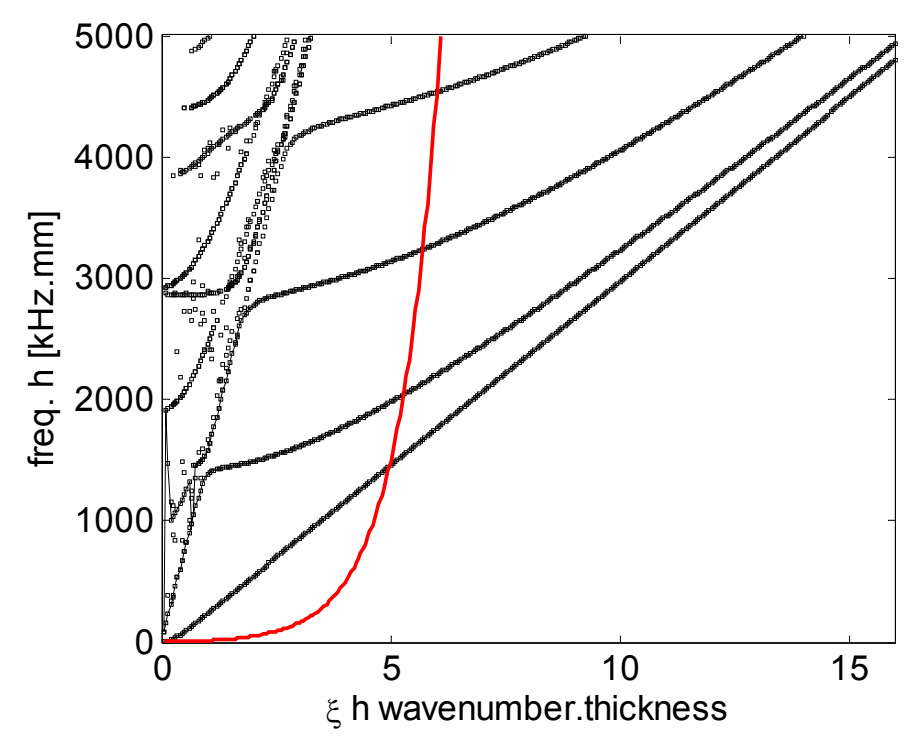

Figure 4. Stiffness matrix solution over the whole domain for U0 fiber composite layer. 
Hence, a combined solution by TMM and SMM is used to obtain a stable and correct solution over the entire solving domain. Figure $5 \mathrm{~b}$ shows the complete frequency - wavenumber solution using combined stiffness transfer matrix method (STMM). Figure 5c,d show phase velocity and group velocity solutions, respectively.

(a)
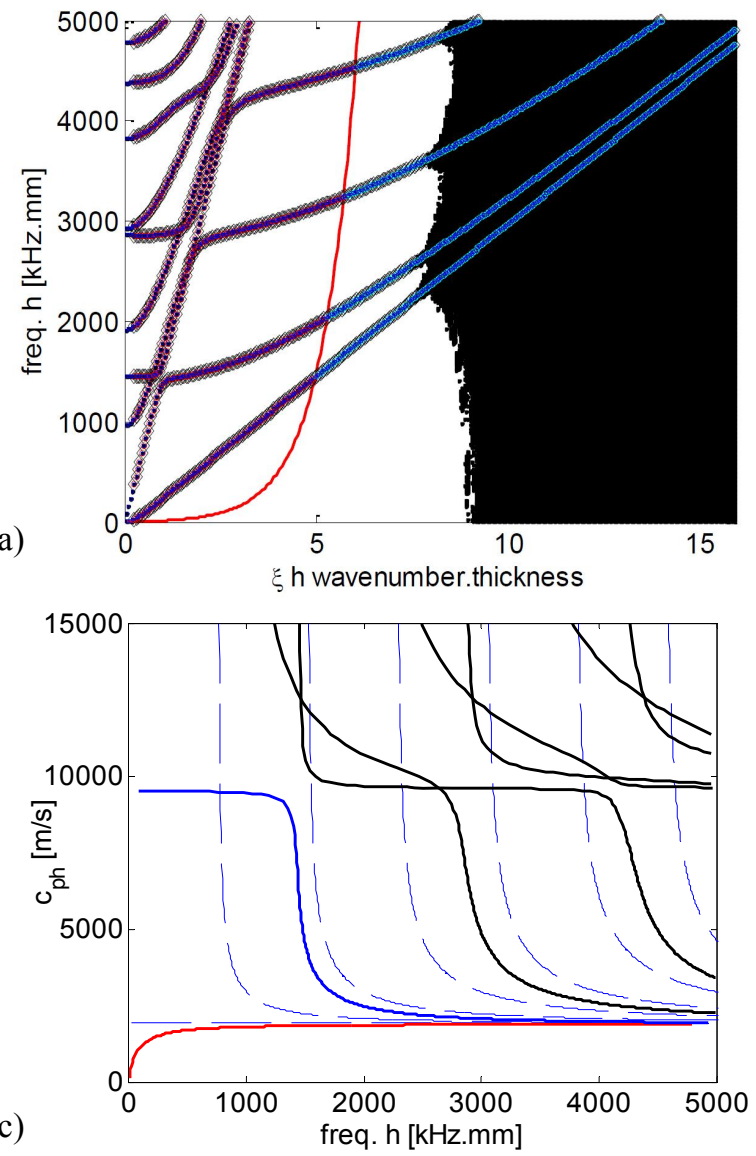

(b)
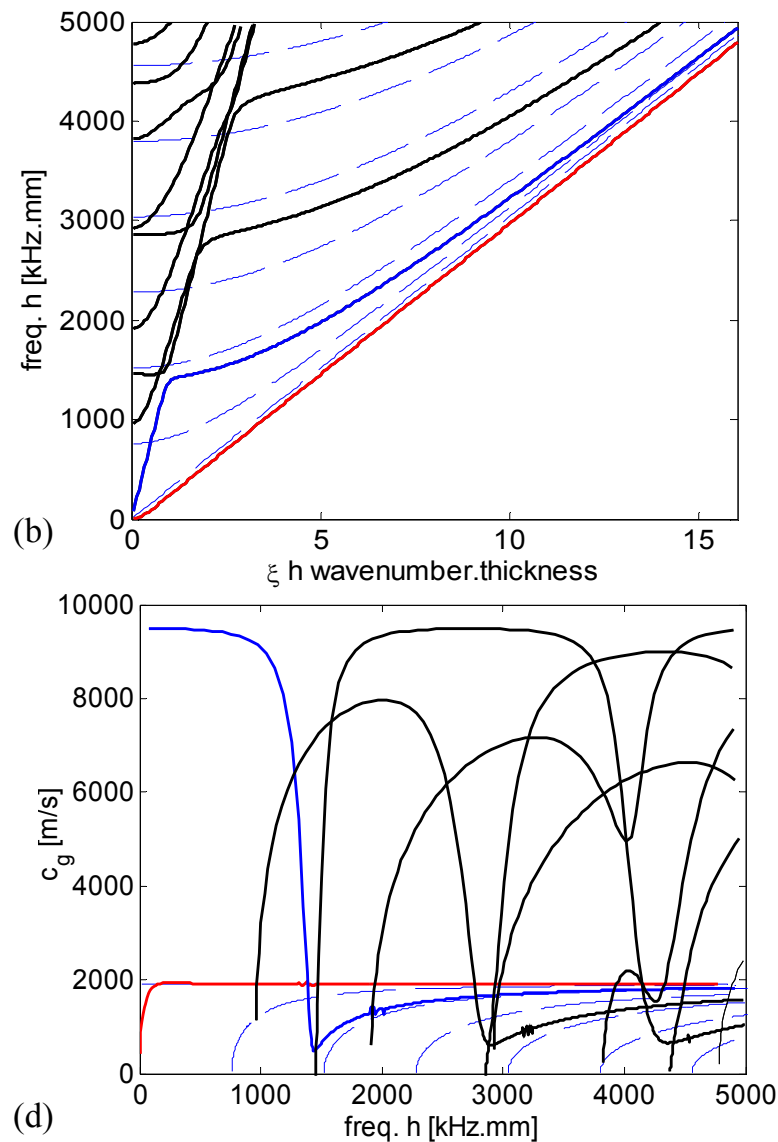

Figure 5. Unidirectional fiber composite lamina, (a) instability of TMM and mode tracking between TMM solution, then SMM solution, (b) complete solution using STMM, (c) complete phase velocity solution using STMM, (d) complete group velocity solution using STMM. —_ Lamb wave solution, - - - Shear horizontal wave solution 


\subsection{Unidirectional fiber composite lamina U45}

Similar analysis was performed for unidirectional CFRP lamina with fibers at $45^{\circ}$ angle from wave propagation direction. Figure 6a shows the instability of TMM and the combined solution of TMM (to the left of the exponential function) and SMM (to the right of the exponential function). Figure $6 \mathrm{~b}$ shows the complete STMM frequency wavenumber solution. Figure $6 \mathrm{c}$,d show the phase velocity solution of TMM and the STMM, respectively. Figure 6 e,f show the group velocity solution of TMM and STMM, respectively.
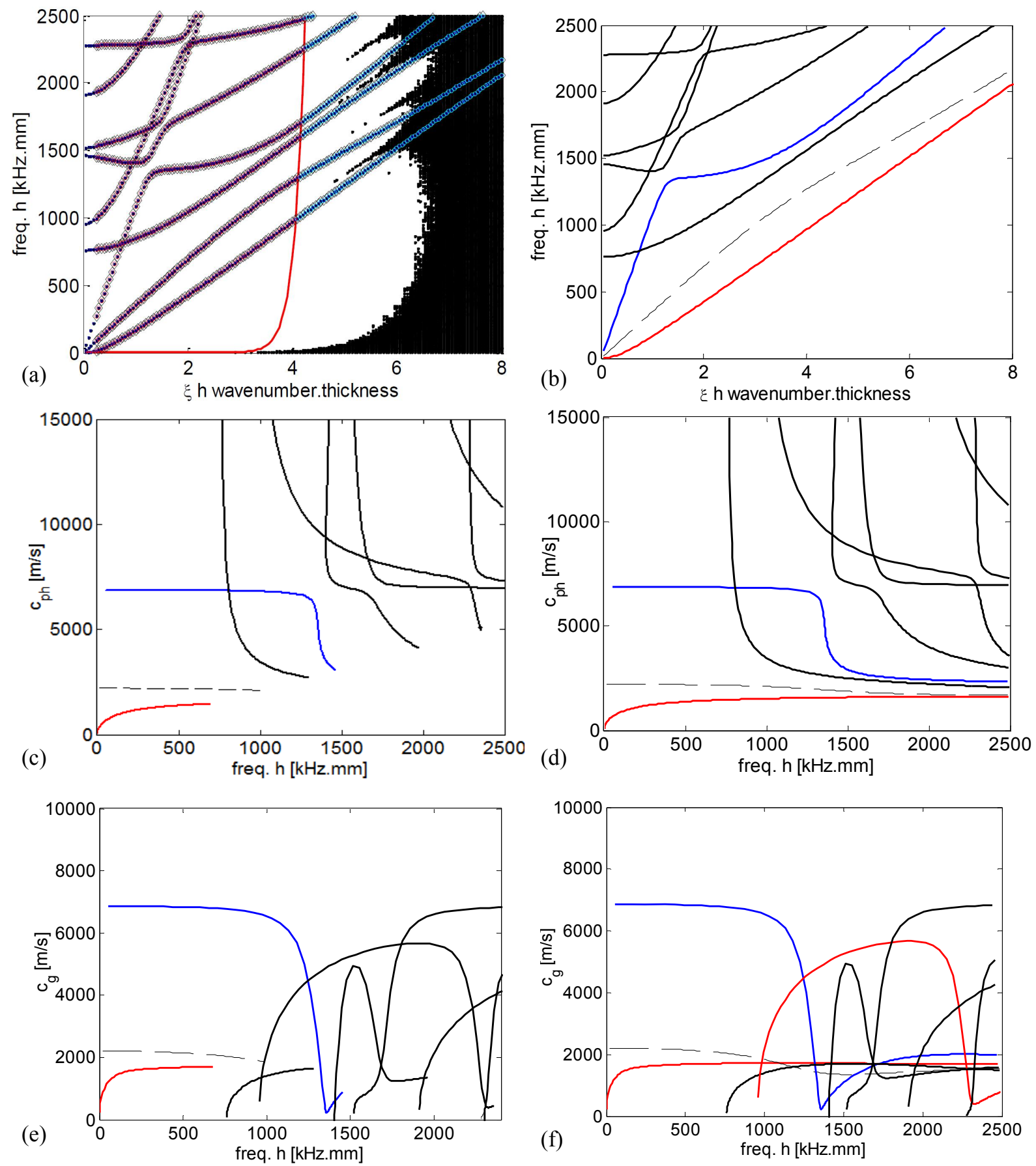

Figure 6 Unidirectional fiber composite lamina with $45^{\circ}$ fibers. __ Lamb wave solution, - - - Shear horizontal wave solution 


\subsection{Cross ply fiber composites $[0 / 90]$}

The last case study is a cross ply CFRP composite laminate [0/90], the layer of $0^{\circ}$ fibers has the same stiffness coefficients of previous unidirectional CFRP. The layer of the $90^{\circ}$ fibers has stiffness coefficients that can be calculated by transformation matrix. Figure 7a shows the frequency - wavenumber roots of the final solution. Figure $7 \mathrm{~b}$ shows the separated modes in frequency - wavenumber domain. Figure $7 \mathrm{c}$, d show phase velocity and group velocity solution respectively. Results were verified by GMM using DISPERSE, and SAFE using GUIGUW.
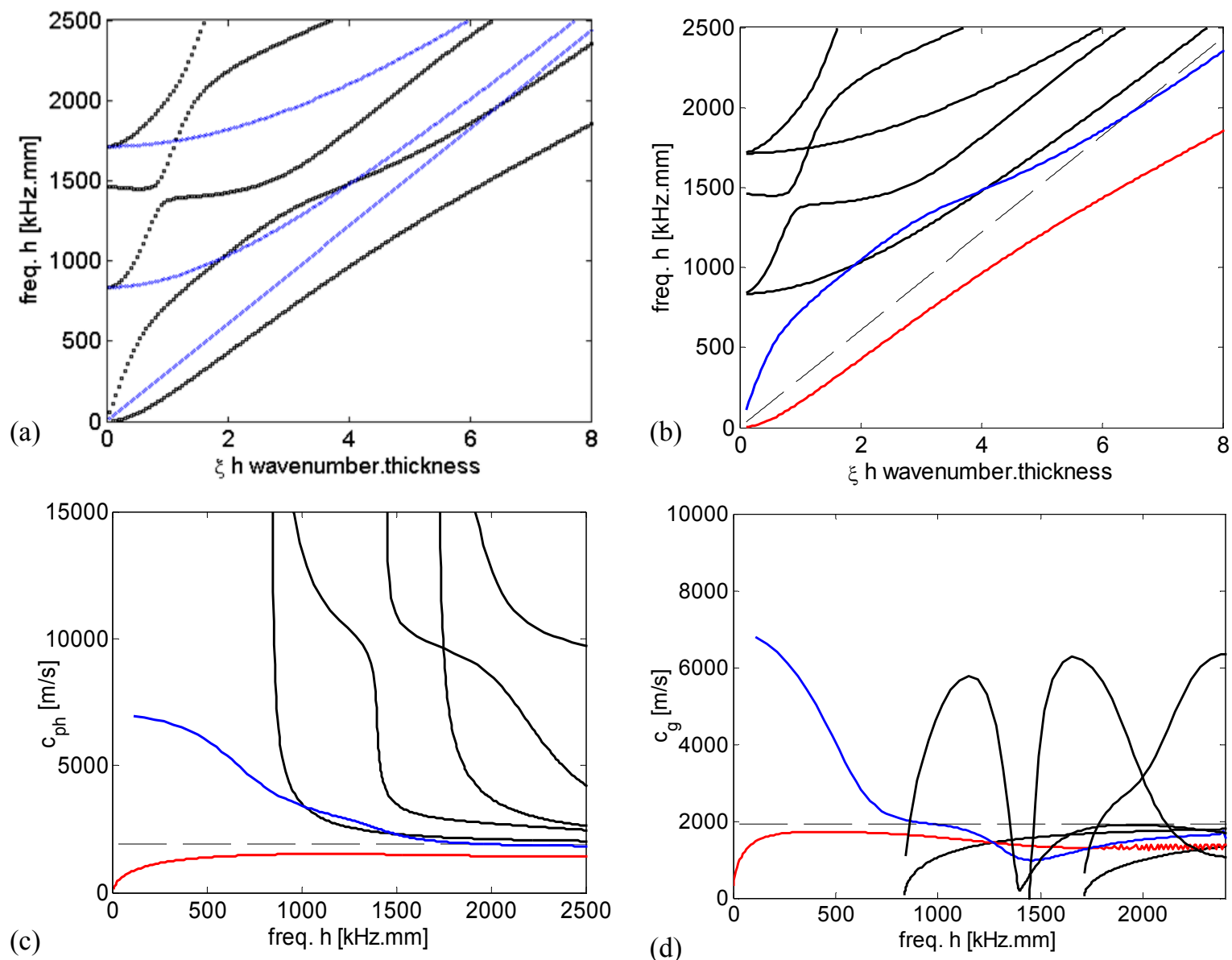

Figure 7. Cross ply fiber composite laminate [0/90],__ Lamb wave solution, - - - Shear horizontal wave solution

\section{SUMMARY AND CONCLUSIONS}

Different algorithms for calculating dispersion wave speeds in composites have been developed over the years, the paper briefly discusses them. This work focused on the transfer matrix method (TMM) and the efforts for generating a stable robust algorithm. TMM is a reliable technique for wave propagation analysis in layered media; its advantage is that it condenses the multi-layered system into equations relating the top and the bottom surfaces of the multilayered domain. TMM suffers instability at high frequency-thickness products. There have been some studies proposing reformulation of TM equations to avoid this problem. The method is based on using stiffness matrix method (SMM) instead of TMM. In this paper, the mathematical formulation was discussed in detail for ease of coding it. It was shown that SMM gives a stable solution at the high frequency- thickness products. But SMM does not give correct roots pattern at low wavenumber and low frequency domain. Hence, a combined stiffness transfer matrix method (STMM) is used to obtain correct and stable results over the entire domain of interest. 


\section{ACKNOWLEDGMENTS}

Support by Air Force Office of Scientific Research, \#FA9550-11-1-0133, Dr. David Stargel, Program Manager, is thankfully acknowledged. The authors would like to thank Prof. Mike Lowe from Imperial College of London, Prof. Ivan Bartoli from Drexel University, and Prof. Alessandro Marzani from University of Bologna for their help in providing some dispersion curves simulations using computer packages they developed. Thanks to Prof. Stanislav Rokhlin, Prof. Evgeny Glushkov, and Prof. Natalia Glushkov for their valuable comments

\section{REFERENCES}

1. Rose, J. L., Ultrasonic Waves in Solid Media, Cambridge University Press, New York, (1999).

2. Kessler, S., Spearing, S., Soutis, C., "Damage detection in composite materials using Lamb wave methods," Smart Materials and Structures, 11, 269-278 (2002).

3. Su, Z., Ye, L., Lu, Y., "Guided Lamb waves for identification of damage in composite structures: A review." J. of Sound and Vibration, 295, 3-5, 753-780 (2006).

4. Raghavan, A., Cesnik, C., "Review of guided-wave structural health monitoring." Shock and Vibration Digestion, 39, 91-114 (2007).

5. Yeum, C. M., Sohn, H., Lim, H. J., Ihn, J. B., "Reference-free delamination detection using Lamb waves.” Structural Control and Health Monitoring, DOI: 10.1002/stc.1594, 1-10 (2013).

6. Viktorov, I. A., [Rayleigh and Lamb waves - physical theory and applications], Plenum Press, New York, (1967).

7. Graff, K. F., Wave motion in elastic solids, Dover Publications Inc., New York, (1991).

8. Giurgiutiu, V., Structural Health Monitoring with Piezoelectric Wafer Active Sensors, Elsevier Academic Press, Amsterdam, (2008).

9. Lamb, H., “On Waves in an Elastic Plate." Proc. R. Soc. A, 93, 293-312 (1917).

10. Conry, M. "Notes on wave propagation in anisotropic elastic solids" http://homepage.tinet.ie/ mjconry/anisotropic.pdf, (2005).

11. Towfighi, S., Kundu, T., Ehsani, M., "Elastic wave propagation in circumferential direction in anisotropic cylindrical curved plates." J. Applied Mechanics, 69, 283-291 (2002).

12. Mal, A. K., "Wave Propagation in layered composite laminates under periodic surface loads." Wave Motion, 10, 257-266 (1988).

13. Smelyanskiy, V. N., Hafiychuk, V., Luchinsky, D. G., Tyson, R., Miller, J., Banks, C., "Modeling wave propagation in Sandwich Composite Plates for Structural Health Monitoring." Annual Conference of the Prognostics and Health Management Society, 1-10 (2011).

14. Banerjee, S., Pol, C. B., "Theoretical modeling of guided wave propagation in a sandwich plate subjected to transient surface excitations." International Journal of Solids and Structures, 49, 3233-3241 (2012).

15. Nayfeh, A. H., Wave Propagation in Layered Anisotropic Media, Elsevier, New York, (1995).

16. Santoni, G., Fundamental Studies in the Lamb-Wave Interaction Between Piezoelectric Wafer Active Sensor and Host Structure During Structural Health Monitoring, Electronic Theses and Dissertations, Dissertation, University of South Carolina, (2010).

17. Schmidt, H., Jensen, F. B., "A full wave solution for propagation in multilayered viscoelastic media with application to Gaussian beam reflection at fluid-solid interfaces." Journal Acoust. Soc. America, 77 (3), 813-825, (1985).

18. Rokhlin, S., Chimenti, D., Nagy, P., [Physical Ultrasonics of Composites], Oxford University Press, (2011).

19. Thomson, W., "Transmission of elastic waves through a stratified solid medium." journal of Applied Physics, 21, 8993 (1950).

20. Haskell, N., "The dispersion of surface waves on multi-layered media." Bulletin of the Seismological Society of America, 43 (1), 17-34 (1953).

21. Lowe, M., "Matrix techniques for modeling ultrasonic waves in multilayered media," Ultrasonics Ferroelectrics and Frequency Control IEEE Transactions, 42 (4), 525-542 (1995). 
22. Knopoff, L. A, "matrix method for elastic wave problems." Bulletin of the Seismological Society of America 54, 431438 (1964).

23. Gavric, L., "Computation of Propagative Waves in Free Rail Using a Finite Element Technique." Journal of Sound and Vibration, 185 (3), 531-543 (1995).

24. Sorohan, S., Constantin, N., Gavan, M., Anghel, V., "Extraction of dispersion curves for waves propagating in free complex waveguides by standard finite element codes." Ultrasonics, 51, 503-515 (2011).

25. Hayashi, T., Song, W.J., Rose, J. L., "Guided wave dispersion curves for a bar with an arbitrary cross-section, a rod and rail example." Ultrasonics, 41, 175-183 (2003).

26. Bartoli, I., Marzani, A., Lanza di Scalea, F., Viola, E., "Modeling Wave Propagation in Damped Waveguides of Arbitrary Cross-section." J. Sound \& Vibration, 295, 685-707 (2006).

27. Delsanto, P. P., Schechter, R. S., Mignogna, R. B., "Connection machine simulation of ultrasonic wave propagation in materials III: The three-dimensional case." Wave Motion, 26 (4), 329-339 (1997).

28. Nadella, K. S., Cesnik, C. E., "Local interaction simulation approach for modeling wave propagation in composite structures." CEAS Aeronautical Journal, 4 (1), 35-48 (2013).

29. Ruzzene, M., Jeong, S. M., Michaels, T. E., Michaels, J. E., Mi, B., "Simulation and measurement of ultrasonic waves in elastic plates using laser vibrometry." Review of progress in Quantitative Non-destructive, Green Bay, Wisconsin, 172-179 (2005).

30. Monnier, T., "Lamb Waves-based Impact Damage Monitoring of a Stiffened Aircraft Panel using Piezoelectric Transducers." J. Int. Mat. Sys. and Structures, 17, 411-421 (2006).

31. Wang, L., Rokhlin, S. I., "Stable reformulation of transfer matrix method for wave propagation in layered anisotropic media." J. Ultrasonics, 39, 407-418 (2001).

32. Jones, R., Mechanics of composite materials, Taylor \& Francis, London, (1999).

33. Pavlakovic, B., Lowe, M., DISPERSE Manual, Imperial College, London, (2003). 